\title{
Evidências sobre a terapia cognitivo- comportamental no tratamento de obesos com transtorno da compulsão alimentar periódica
}

\author{
Evidence of cognitive-behavioral therapy in the treatment of obese patients with \\ binge eating disorder
}

\author{
Mônica Duchesne ${ }^{1}$, José Carlos Appolinário ${ }^{1}$, Bernard Pimentel Rangé ${ }^{2}$, Silvia Freitas ${ }^{1}$, Marcelo \\ Papelbaum $^{1}$, Walmir Coutinho ${ }^{3}$
}

\begin{abstract}
${ }^{1}$ Instituto de Psiquiatria, Universidade Federal do Rio de Janeiro (UFRJ), Rio de Janeiro, RJ. Grupo de Obesidade e Transtornos Alimentares, Instituto Estadual de Diabetes e Endocrinologia, Rio de Janeiro, RJ. ${ }^{2}$ Instituto de Psicologia, UFRJ, Rio de Janeiro, RJ. ${ }^{3}$ Grupo de Obesidade e Transtornos Alimentares, Instituto Estadual de Diabetes e Endocrinologia, Rio de Janeiro, RJ.
\end{abstract}

\section{Resumo}

Objetivo: Avaliar as evidências sobre a eficácia da terapia cognitivo-comportamental no tratamento de obesos com transtorno da compulsão alimentar periódica.

Método: Nesta revisão, foram incluídos ensaios clínicos e metanálises publicados entre janeiro de 1980 e fevereiro de 2006, em todas as línguas. Foram excluídos estudos que investigassem a eficácia da terapia cognitivo-comportamental com uso concomitante de medicação, terapia cognitivo-comportamental no formato de manuais de auto-ajuda, relatos ou séries de casos e cartas ao editor. As bases eletrônicas de dados consultadas foram: MEDLINE, PsycINFO, Embase, LILACS e Cochrane Library. A estratégia de busca incluiu também a checagem manual das referências bibliográficas dos artigos selecionados e de capítulos de livros sobre o tema.

Resultados: Foram encontrados dois ensaios clínicos abertos e 15 controlados. O desfecho primário na maioria desses estudos é a compulsão alimentar. No geral, os ensaios clínicos avaliados sugerem que o uso da terapia cognitivo-comportamental resulta numa melhora significativa da compulsão alimentar e dos sintomas psicopatológicos associados ao transtorno da compulsão alimentar periódica, sem resultar em perda de peso substancial.

Conclusões: As evidências disponíveis sugerem que a terapia cognitivo-comportamental é um método de tratamento eficaz para o transtorno da compulsão alimentar, em relação aos componentes psicológicos dessa condição. Entretanto, sua eficácia na redução do peso corporal e na manutenção dos seus efeitos no longo prazo ainda precisa ser melhor investigada.

Descritores: Terapia cognitivo-comportamental, tratamento, psicoterapia, transtorno da compulsão alimentar periódica, compulsão alimentar, obesidade.

\begin{abstract}
Objective: To investigate evidence of the efficacy of cognitive-behavioral therapy in the treatment of obese patients with binge eating disorder.

Method: This review included clinical trials and meta-analyses published in all languages from January 1980 to February 2006. Studies assessing the efficacy of cognitive-behavioral therapy associated with medication, cognitive-behavioral therapy in self-help manuals, case reports or series and letters to editors were excluded. The following electronic databases were used: MEDLINE, PsycINFO, Embase, LILACS and Cochrane Library. Search strategies also included consulting the references of selected articles and chapters of specialized books.

Results: Two open and 15 controlled clinical trials were included. The primary outcome in most studies was binge eating. In general, the clinical trials suggest that cognitive-behavioral therapy results in significant improvement in binge eating and other psychopathological symptoms related to binge eating disorder. However, no substantial weight loss was reported.
\end{abstract}

Correspondência:

Mônica Duchesne, Rua Marquês de São Vicente 124/239, Gávea, CEP 22451-040, Rio de Janeiro, RJ. E-mail: mduchesne@rionet.com.br Copyright $\odot$ Revista de Psiquiatria do Rio Grande do Sul - SPRS

Recebido em 10/07/2006. Aceito em 30/01/2007. 
Conclusions: Available evidence suggests that cognitive-behavioral therapy is an effective intervention method for psychological aspects of binge eating disorder, although its efficacy in body weight reduction and long-term maintenance of results still needs further investigation. Keywords: Cognitive-behavioral therapy, treatment, psychotherapy, binge eating disorder, binge eating, obesity.

\section{Introdução}

O transtorno da compulsão alimentar periódica (TCAP) é caracterizado por episódios de compulsão alimentar recorrentes na ausência dos comportamentos compensatórios inadequados para evitar o ganho de peso observados na bulimia nervosa (BN). Durante o episódio de compulsão alimentar, há um sentimento de falta de controle sobre o comportamento associado à ingestão de grandes quantidades de alimento, mesmo que o indivíduo esteja sem fome, levando a um grande desconforto. Esse episódio é sucedido por um intenso mal-estar subjetivo, caracterizado por sentimentos de angústia, tristeza, culpa, vergonha e/ou repulsa por si mesmo ${ }^{1}$.

Apesar do peso não ser um critério diagnóstico, o TCAP freqüentemente se associa ao sobrepeso e a diversos graus de obesidade. Enquanto sua prevalência estimada na população em geral pode variar entre $1,8^{2}$ e $4,6 \%{ }^{3}$, aproximadamente $30 \%$ dos indivíduos obesos que procuram tratamento para emagrecer apresentam esse transtorno ${ }^{2,3}$, tendo sido observada uma associação positiva entre a presença da compulsão alimentar e o aumento da adiposidade ${ }^{3,4}$.

Os obesos com TCAP, quando comparados aos sem esse transtorno, apresentam mais sintomas psicopatológicos gerais ou alimentares ${ }^{4}$. Em relação ao primeiro grupo, foram identificados maiores níveis de perfeccionismo ${ }^{5}$, impulsividade ${ }^{6}$, ansiedade ${ }^{6}$ e isolamento social $^{6}$, além de uma maior vulnerabilidade à depressão ${ }^{5,7-}$ ${ }^{9}$. Adicionalmente, foi relatada uma pior qualidade de vida ${ }^{10} \mathrm{e}$ uma pior auto-estima ${ }^{7}$ nesse grupo de indivíduos. Com relação aos sintomas psicopatológicos específicos do TCAP, foram observadas atitudes mais disfuncionais relacionadas à alimentação e ao peso, além de uma extrema preocupação e insatisfação com a forma corporal $^{4,5,8,11,12}$. Adicionalmente, evidências sugerem que o subgrupo de obesos com TCAP apresenta uma pior resposta aos tratamentos que objetivam emagrecimento ${ }^{5,8,10,13,14}$, um maior número de tentativas malsucedidas de adesão a dietas ${ }^{3,10}$, uma maior dificuldade para perder peso $^{5,8,10,13,14}$, uma pior manutenção do peso perdido ${ }^{8,10,13,14}$ e uma maior taxa de abandono dos tratamentos ${ }^{8}$.

A terapia cognitivo-comportamental (TCC) foi considerada a forma de intervenção psicoterápica mais investigada no TCAP através de ensaios clínicos randomizados ${ }^{15} \mathrm{e}$ tem sido crescentemente utilizada em diversos centros especializados no tratamento dos transtornos alimentares. Ela baseia-se no pressuposto de que um sistema disfuncional de crenças está associado ao desenvolvimento e manutenção do TCAP. Conseqüentemente, a modificação de padrões distorcidos de raciocínio e a reestruturação de crenças supervalorizadas associadas ao peso e à imagem corporal são focos primários do tratamento, sendo utilizadas várias técnicas cognitivas com essa finalidade.

Além de técnicas cognitivas, a TCC também emprega técnicas comportamentais para ajudar na modificação dos hábitos alimentares. Como exemplos dessas técnicas, podem ser citadas a automonitoração (observação sistemática e registro dos alimentos ingeridos e das circunstâncias associadas), as técnicas para controle de estímulos (que envolvem a identificação das situações que favorecem a ocorrência da compulsão alimentar e o desenvolvimento de um estilo de vida que minimize o contato do paciente com essas situações) e o treinamento em resolução de problemas, que ajuda o paciente a desenvolver estratégias alternativas para enfrentar suas dificuldades sem recorrer à alimentação inadequada. Em geral, a TCC também focaliza estratégias para prevenção de recaídas ${ }^{16-19}$. Por fim, embora existam algumas diferenças no modo como ela foi empregada em diferentes ensaios clínicos, os programas utilizados nesses estudos são semi-estruturados, geralmente implementados no formato de grupo (com aproximadamente 10 pacientes) e com a duração média de 12 sessões de 90 minutos.

O presente artigo faz uma revisão crítica da literatura disponível sobre a eficácia da TCC aplicada a obesos com TCAP, além de discutir possíveis modificações nos protocolos de tratamento atualmente utilizados.

\section{Método}

Foi realizada uma pesquisa da literatura utilizandose as bases de dados eletrônicas MEDLINE, PsycINFO, Embase, LILACS e Cochrane Library. Foram utilizadas as seguintes categorias de termos MeSH (Medical Subject Headings): binge, binge eating, binge eating disorder, bulimia nervosa, obesity e body image versus cognitivebehavior therapy, cognitive therapy, behavior therapy, psychological support e relapse. Para complementar a estratégia de busca, foi realizada a checagem manual das referências bibliográficas dos artigos selecionados, buscando artigos e capítulos de livros de interesse sobre o tema, com o objetivo final de localizar textos pertinentes que não haviam sido encontrados através da busca eletrônica. Foram incluídos ensaios clínicos abertos e 
controlados e metanálises publicadas entre janeiro de 1980 e fevereiro de 2006, em todas as línguas, que avaliassem a eficácia da TCC no tratamento do TCAP. Foram excluídos estudos que investigassem a eficácia da TCC em associação com fármacos, TCC no formato de manuais de auto-ajuda, cartas ao editor e relatos ou séries de casos.

O Manual Diagnóstico e Estatístico de Transtornos Mentais (DSM-IV) ${ }^{1}$ especifica uma freqüência de compulsão alimentar de pelo menos 2 dias na semana, durante um período mínimo de 6 meses, para estabelecer o diagnóstico de TCAP. Entretanto, os critérios do DSM-IV só foram estabelecidos em 1994, e, conseqüentemente, a duração, a freqüência e a gravidade dos aspectos comportamentais associados à compulsão alimentar nos obesos pesquisados nos ensaios clínicos variaram nos primeiros estudos. Portanto, esta revisão inclui artigos com pacientes com TCAP, TCAP subclínico e obesos com BN não-purgativa. Essas últimas categorias diagnósticas são consideradas correlatos de TCAP, pois incluem, em sua maioria, pacientes que na DSM-IV vieram a ser conceituados como portadores desse diagnóstico.

\section{Resultados}

Foram encontradas 348 publicações que envolviam temas associados à TCC no TCAP. Após uma avaliação dos resumos dessas publicações, 84 artigos foram inicialmente selecionados e solicitados na sua íntegra. Após a leitura desses artigos, 21 estudos em inglês foram incluídos nesta revisão, por preencherem os critérios de inclusão: dois estudos abertos, 15 estudos controlados e uma metanálise. A seguir, descreveremos os resultados dos ensaios clínicos utilizando essa forma de psicoterapia no TCAP, focalizando a eficácia da TCC nos sintomas psicopatológicos associados e característicos do TCAP e no peso corporal.

\section{Sintomas psicopatológicos característicos do TCAP}

\section{Compulsão alimentar}

As primeiras avaliações da eficácia da TCC adaptada para obesos com compulsão alimentar foram realizadas em pacientes com BN de subtipo não-purgativo que apresentavam episódios de compulsão alimentar duas vezes por semana, por pelo menos 6 meses, a maioria deles com sobrepeso ou obesidade ${ }^{20,21}$. Por exemplo, em um estudo controlado randomizado com 44 pacientes (índice de massa corporal - IMC $=32,6$ ), Telch et al. ${ }^{20}$ observaram 94\% de redução da freqüência da compulsão alimentar após 10 sessões de TCC, em comparação a 9\% dos pacientes alocados em uma lista de espera.
Em pacientes obesos com TCAP, estudos abertos sugerem que a TCC no formato de grupo favorece uma redução da freqüência da compulsão alimentar, variando entre $80^{22}$ e $91 \%{ }^{23}$. Adicionalmente, estudos controlados randomizados também relataram reduções significativas na freqüência da compulsão ao final da TCC ${ }^{11,12,24-27}$. Agras et al. ${ }^{24}$ realizaram um estudo comparativo entre TCC e terapia interpessoal (TI), um formato de terapia que focaliza o aumento da capacidade para lidar com dificuldades interpessoais e com os papéis sociais no tratamento do $\mathrm{TCAP}^{21}$. Inicialmente, 50 pacientes foram randomizados para TCC ou para uma lista de espera, tendo sido observada uma taxa de $55 \%$ de remissão após 12 sessões de TCC (e 9\% na lista de espera). Os pacientes que ainda apresentavam compulsão no final do tratamento receberam 12 sessões adicionais de TI, e os demais receberam 12 sessões de terapia comportamental (TC) focalizada na perda de peso. A associação de TI ou de TC não resultou em melhora adicional da compulsão alimentar. Em um outro ensaio clínico, Eldredge et al. ${ }^{12}$ observaram 50\% de redução da freqüência após 12 sessões de tratamento e 19,8\% numa lista de espera. Um dos braços desse estudo envolveu oferecer aos pacientes que não melhoraram 12 sessões adicionais de TCC. Ao final das 24 sessões, foi observada uma redução de $68 \%$ da compulsão alimentar e um aumento da taxa de remissão (2/3 dos pacientes).

Alguns estudos controlados randomizados compararam a eficácia da TCC isoladamente com a TCC associada a fármacos ${ }^{7,27-29}$. Esses estudos demonstraram reduções significativas da compulsão alimentar após a TCC, sem benefício adicional com o uso de desipramina ${ }^{7}$, fluoxetina ${ }^{27-29}$ ou fluvoxamina ${ }^{27}$. Grilo et al. ${ }^{29}$ randomizaram 108 pacientes para quatro condições: TCC no formato individual combinada com fluoxetina, TCC combinada com placebo, fluoxetina ou placebo. Eles observaram taxas de remissão significativamente mais altas nos grupos com TCC, sem melhora significativa para o grupo em uso de fluoxetina ou placebo sem TCC. As taxas de remissão foram: $29 \%$ (fluoxetina), 30\% (placebo), 55\% (TCC combinada com fluoxetina) e 73\% (TCC combinada com placebo). Em um outro estudo, também utilizando TCC no formato individual, Devlin et al. ${ }^{28}$ comparam a eficácia de adicionar fluoxetina ou TCC a um programa de TC. Foi observada uma melhora significativa da compulsão alimentar como resultado da adição da TCC com 62\% dos pacientes que receberam TCC atingindo remissão (e 33\% no grupo-controle). Nenhuma diferença significativa nas taxas de remissão foi observada com a adição de fluoxetina em comparação ao placebo.

Kenardy et al. ${ }^{30}$ estudaram a eficácia da TCC em pacientes obesos com diabetes tipo 2 que apresentavam compulsão pelo menos uma vez por semana. Comparando a TCC com um tratamento baseado nos 
princípios de autoconhecimento e auto-aceitação de Rogers $^{31}$, eles demonstraram que ambos os tratamentos foram eficazes na redução da freqüência da compulsão alimentar, com uma taxa de remissão de $47 \%$ no final da TCC e de $29 \%$ no tratamento alternativo. Adicionalmente, observaram que a melhora na compulsão alimentar associou-se a uma melhora do controle glicêmico.

Além da redução da freqüência dos episódios de compulsão alimentar, a diminuição da gravidade da compulsão alimentar foi avaliada em vários estudos através da Escala de Compulsão Alimentar Periódica ${ }^{32}$. Estudos controlados randomizados identificaram uma diminuição da gravidade da compulsão alimentar como resultado da TCC, comparando-a à $\mathrm{TC}^{33}$ e a listas de espera $^{12,24}$. Portanto, os ensaios clínicos anteriormente descritos sugerem que a TCC resulta em redução significativa da freqüência e da gravidade da compulsão alimentar.

Atitudes associadas à alimentação e à forma corporal

Além da compulsão alimentar, alguns ensaios clínicos investigaram outros comportamentos e atitudes disfuncionais associados à alimentação, peso e forma corporais em obesos com TCAP ${ }^{11,12,22-27,29,30,33-35}$. Um dos aspectos estudados é a tentativa (em geral, malsucedida) de restringir a alimentação de forma inadequada para obter emagrecimento. Utilizando a subescala Cognitive Restraint do Three-Factor Eating Questionnaire ${ }^{36}$, Smith et al. ${ }^{23}$ não observaram melhora na tendência para restringir a alimentação em nove pacientes que receberam 16 sessões de TCC. Utilizando a mesma subescala, Eldredge et al. ${ }^{12}$, Agras et al. ${ }^{24}$ e Grilo et al..$^{29}$ também não observaram alteração nos níveis de restrição alimentar ao compararem os resultados da TCC em grupo com uma lista de espera ou com um grupo em uso de fluoxetina. Entretanto, alguns estudos controlados randomizados evidenciaram uma melhora da restrição e a adoção de atitudes mais flexíveis em relação à alimentação ${ }^{11,26,27,33}$. Por exemplo, Ricca et al. ${ }^{27}$ alocaram randomicamente 107 pacientes em cinco condições de tratamento: TCC isoladamente, TCC combinada com fluoxetina, TCC combinada com fluvoxamina, fluoxetina sem TCC e fluvoxamina sem TCC. Utilizando a subescala Restraint da Eating Disorder Examination ${ }^{37}$, eles observaram uma redução significativa dos níveis de restrição alimentar e da preocupação disfuncional com a alimentação nos pacientes submetidos apenas à TCC e à TCC combinada com psicofármacos, não tendo sido relatada melhora nos pacientes que receberam apenas fluoxetina ou fluvoxamina.

Estudos comparativos de TCC com TCC associada à orientação nutricional ${ }^{34,35}$ revelaram uma diminuição significativa da propensão para apresentar compulsão alimentar, avaliada pela subescala "Bulimia” do Eating Disorder Inventory ${ }^{38}$. Adicionalmente, em estudos controlados comparando TCC com lista de espera ${ }^{12,24}$, TCC com $\mathrm{TC}^{7}$ e TCC com fluoxetina ${ }^{29}$, os pacientes relataram um aumento do controle do impulso para comer inadequadamente, avaliado pela subescala Desinhibition do Three-Factor Eating Questionnaire ${ }^{36}$. Stunkard et al. ${ }^{36}$ descreveram que a melhora da pontuação nessa subescala se associa ao aumento efetivo da capacidade para controlar a alimentação quando o indivíduo é exposto a situações emocionalmente estressantes (que poderiam favorecer a ocorrência da compulsão alimentar). Esses dados, somados, são particularmente interessantes, uma vez que, de acordo com Fairburn et al. ${ }^{16}$, Lowe \& Caputo ${ }^{39}$ e Laessle et al. ${ }^{40}$, a diminuição dos níveis de restrição e a melhora da capacidade para controlar o impulso para alimentar-se excessivamente apresentam uma correlação positiva com um maior sucesso no controle da compulsão alimentar e da alimentação de modo geral.

Estudos abertos ${ }^{22,23}$ e controlados ${ }^{11,27,29,30,34,35}$, comparando a TCC com a TI, com uma terapia com base nos princípios de Rogers ou com psicofármacos, relataram uma redução significativa da preocupação extrema com a forma e o peso corporal e um aumento da satisfação com a imagem corporal no final da TCC $^{11,27,29,30,34,35}$. Observou-se superioridade da TCC quando comparada à fluoxetina ${ }^{27,29}$ e à fluvoxamina ${ }^{27} \mathrm{e}$ efeitos similares aos obtidos pelos outros dois formatos de terapia testados. Em alguns ensaios clínicos, a diminuição do grau de insatisfação com a forma corporal ocorreu mesmo na ausência de perda de peso $^{8,22,30}$. Estudos de TCC com obesos sem TCAP ${ }^{41,42}$ também revelaram que a melhora da insatisfação com a imagem corporal não estava correlacionada com o IMC. Esses achados levaram Wolff \& Clark $^{22}$ e Parham ${ }^{43}$ a sugerirem que o grau de satisfação com a imagem corporal pode estar mais correlacionado com o sistema de crenças do indivíduo do que com o grau de obesidade, ressaltando a importância de incluir estratégias para modificação do sistema de crenças nos programas de tratamento, objetivando melhorar a relação do paciente com sua imagem corporal.

A análise dos ensaios clínicos descritos sugere que o uso da TCC resulta numa diminuição significativa da preocupação disfuncional com a alimentação, peso e forma corporal, além de favorecer uma melhora das atitudes associadas à alimentação.

\section{Sintomas psicopatológicos gerais associados ao TCAP}

Alguns ensaios clínicos avaliaram os resultados obtidos pela TCC no funcionamento interpessoal, na auto-estima e nos níveis de depressão e ansiedade dos

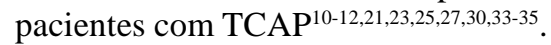


Em um estudo comparativo de TCC com lista de espera, Eldredge et al. ${ }^{12}$ não relataram melhora dos níveis de depressão após 24 sessões de tratamento. Entretanto, um estudo aberto ${ }^{23}$ e vários estudos controlados randomizados ${ }^{25,27,29,33-35}$ com obesos com TCAP descreveram uma melhora significativa nos níveis de depressão no final da TCC. Por exemplo, Ricca et al. ${ }^{27}$ utilizaram o Beck Depression Inventory ${ }^{44}$ e relataram que, embora a TCC não tenha sido primariamente direcionada para a melhora do humor, houve uma redução significativa e equivalente dos níveis de depressão em grupos tratados com TCC e TCC combinada com fluoxetina ou fluvoxamina. Esses autores sugeriram que a redução da compulsão alimentar e a diminuição da insatisfação com a imagem corporal resultantes da TCC podem ter contribuído para a melhora do humor em pacientes obesos com TCAP.

Dois ensaios clínicos evidenciaram que a TCC favorece uma redução dos níveis de ansiedade ${ }^{27,35}$. Entretanto, Fossati et al. ${ }^{34}$ não relataram melhora significativa dos níveis de ansiedade após a TCC em pacientes que receberam TCC isoladamente ou combinada com nutrição. Contudo, houve um decréscimo significativo em um grupo tratado com TCC combinada com orientação nutricional e exercício.

Alguns estudos controlados randomizados indicaram uma melhora nas dificuldades interpessoais $^{11,12,34,35,45}$, na auto-estima ${ }^{11}$ e no funcionamento social como resultado da TCC em grupo. Utilizando o Rosenberg Self-Esteem Questionnaire ${ }^{46}$ e o Inventory of Interpersonal Problems ${ }^{47}$, Eldredge et al. ${ }^{12}$ compararam a TCC com uma lista de espera e reportaram melhora significativa das dificuldades interpessoais. Entretanto, nesse estudo, não foi relatada melhora na auto-estima.

Estudando 34 obesas diabéticas com compulsão alimentar, Kenardy et al. ${ }^{30}$ observaram uma melhora do sentimento de bem-estar subjetivo avaliado pelo Well Being Questionnaire ${ }^{48}$, que foi adaptado para pacientes diabéticos. Em um outro estudo, Marchesini et al. ${ }^{45}$ relataram uma melhora da percepção da qualidade de vida em obesos com TCAP, utilizando o SF-36 ${ }^{49}$. Comparando um programa de TCC seguido por TC com uma lista de espera, eles observaram que todas as subescalas do SF-36 melhoraram significativamente no grupo de TCC e não variaram no grupo-controle. Em um estudo aberto, Wolff \& Clark $^{22}$ reportaram uma melhora significativa na auto-eficácia em 20 pacientes com TCAP após um programa de TCC de 15 semanas.

O conjunto de dados apresentados sugere que, ao final da TCC, há uma melhora das dificuldades interpessoais, do grau de adaptação social, da autoestima, dos níveis de ansiedade e depressão, além de uma melhora na qualidade de vida e do sentimento de bem-estar subjetivo.

\section{Peso corporal}

Quatorze ensaios clínicos investigaram os resultados obtidos pela TCC sobre o peso corporal em pacientes com TCAP. Em dois estudos abertos ${ }^{22,23}$ e 10 estudos controlados ${ }^{11,12,20,21,24-26,28,29,34}$, não foi observada uma redução significativa no peso corporal. Por exemplo, Agras et al. ${ }^{24}$ compararam um programa de TCC com 12 sessões com uma lista de espera e, no final do tratamento, não relataram redução no IMC dos pacientes. Em contrapartida, dois estudos controlados reportaram reduções de peso significativas ${ }^{19,27,30}$. Ricca et al. ${ }^{27}$ observaram uma redução de cinco pontos no IMC de obesos com TCAP que receberam TCC ou TCC combinada com fluoxetina ou fluvoxamina, sem diferenças significativas entre os grupos.

A eficácia de associar orientação nutricional e atividade física à TCC foi avaliada em três estudos controlados randomizados, tendo sido observadas reduções significativas no peso corporal com essa associação $0^{25,34,35}$. Fossati et al. ${ }^{34}$ observaram uma perda média de 1,5 kg em um grupo de TCC combinada com orientação nutricional; de 2,8 kg em um grupo que recebeu TCC, orientação nutricional e atividade física; e não observaram perda de peso no grupo que recebeu apenas TCC (0,3 kg).

Uma outra linha de pesquisa demonstrou que a associação de programas de TC subseqüentemente à TCC favorece o emagrecimento. Os programas de TC não incluem técnicas focalizadas na modificação das idéias supervalorizadas associadas ao peso e formato corporais, mas utilizam as técnicas comportamentais anteriormente citadas na descrição dos programas de TCC. Eles enfatizam as estratégias que favorecem o emagrecimento, incentivam a restrição alimentar e, em alguns estudos, são associados a dietas hipocalóricas. Agras et al. ${ }^{24}$ submeteram os pacientes em remissão de compulsão alimentar após 12 sessões de TCC a um programa de 12 sessões de TC, tendo sido observada uma perda média de 4,1 kg no final da TC.

Ao analisar-se o perfil individual de resposta dos pacientes à TCC, observa-se que a melhora da compulsão alimentar está significativamente correlacionada com o emagrecimento ${ }^{25,33,45}$. Em geral, os pacientes que apresentam remissão ou redução significativa da freqüência da compulsão alimentar perdem peso, e, em contrapartida, aqueles que não obtêm redução usualmente ganham peso ${ }^{11,14,23,24,26,28,29}$. Agras et al. $^{26}$ relataram que a remissão total da compulsão alimentar correlacionou-se com a perda de peso com $92 \%$ de probabilidade, enquanto todos os outros níveis de redução estavam associados a $60 \%$ ou menos de chance de perda de peso.

Os resultados da TCC na compulsão alimentar e no peso corporal são apresentados na Tabela 1. 


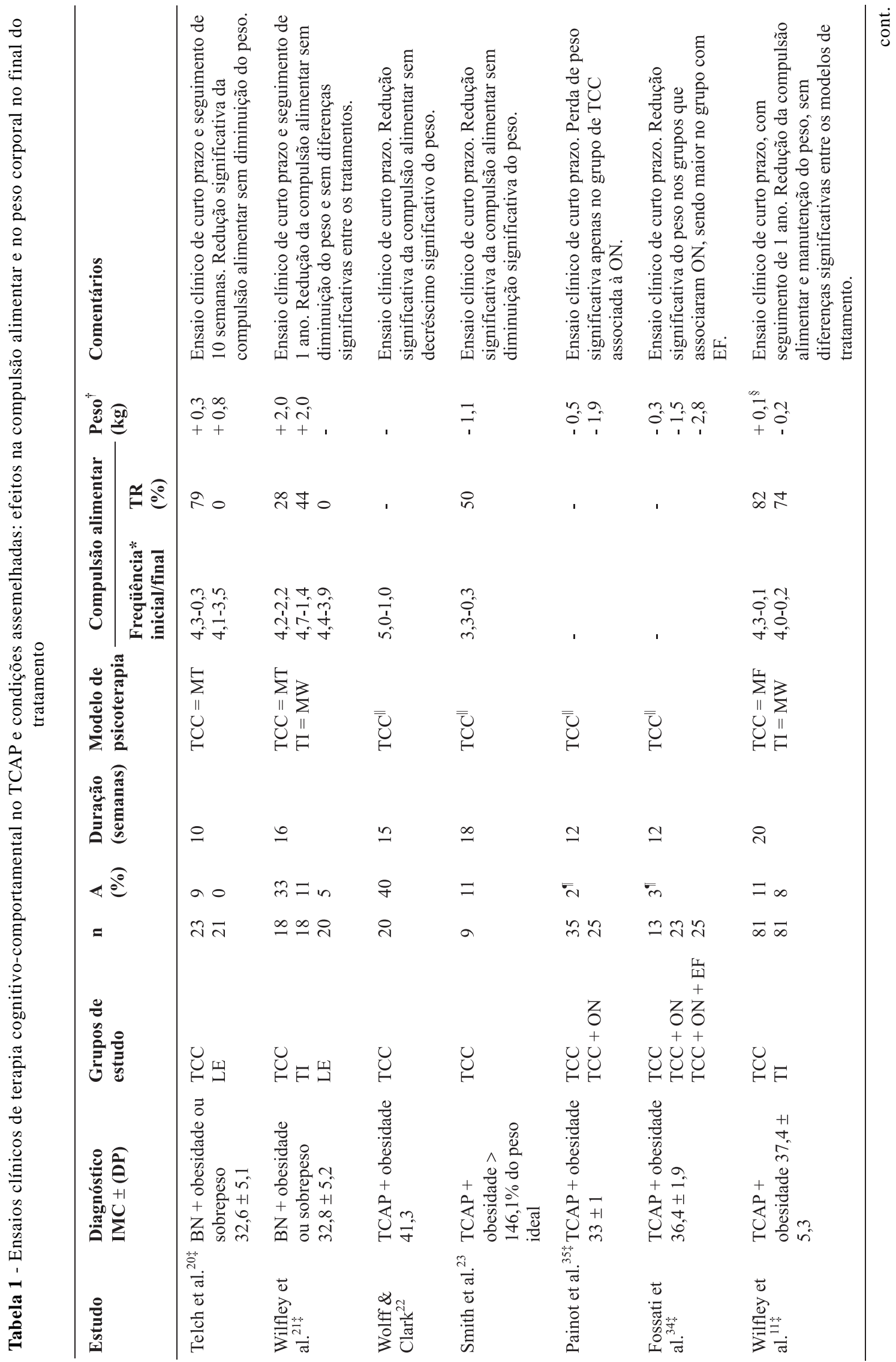




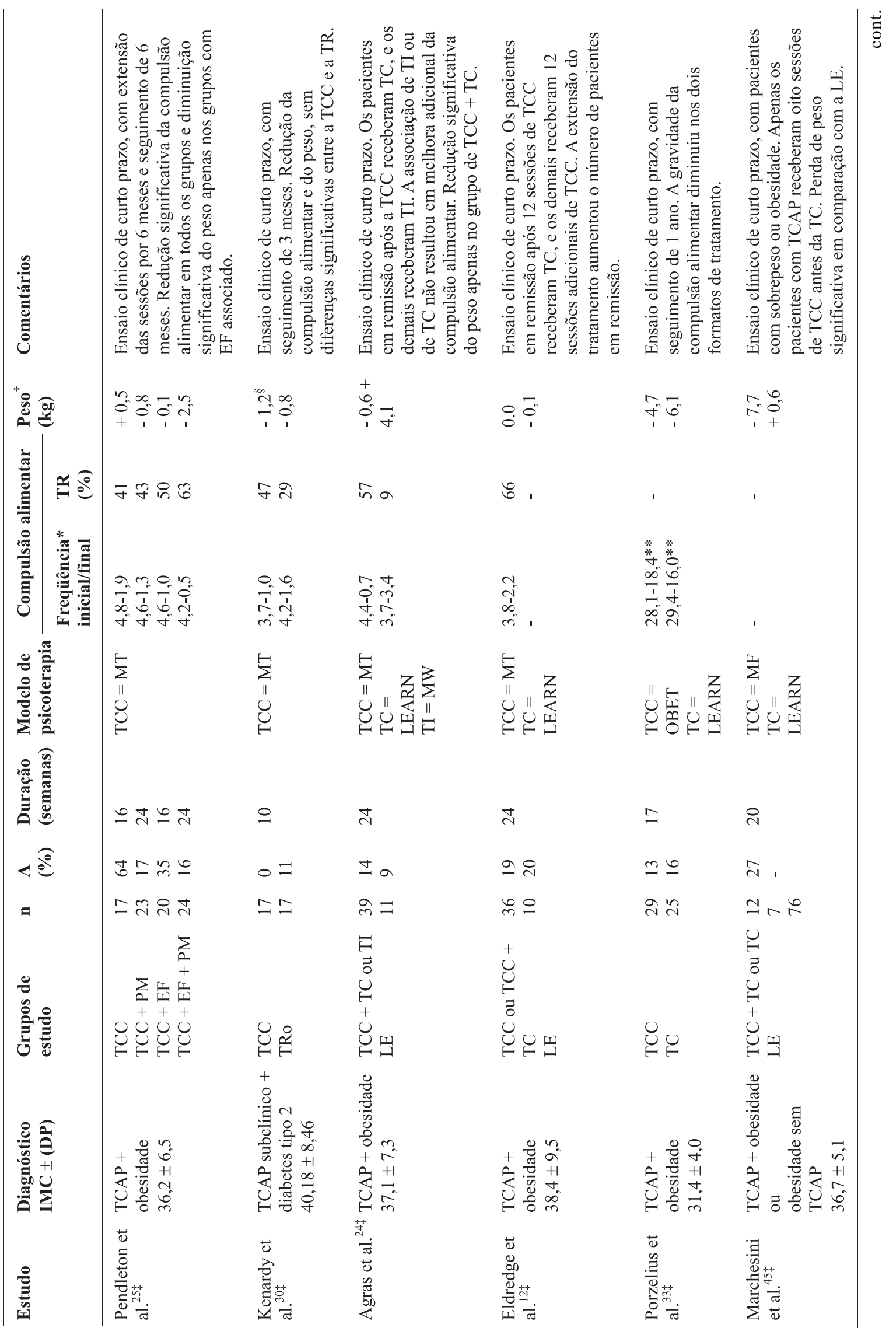




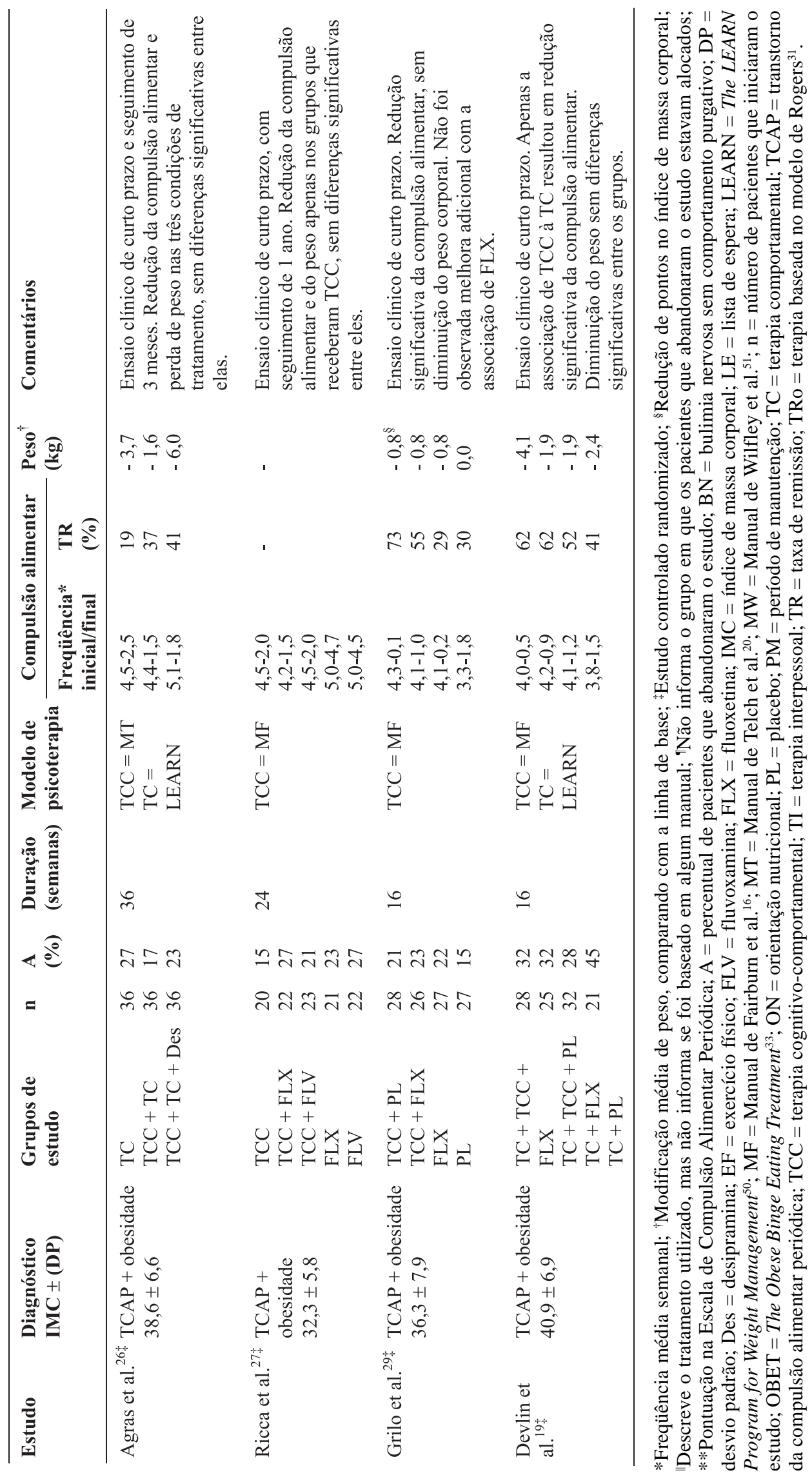




\section{Manutenção a longo prazo dos resultados obtidos com a TCC}

Alguns estudos de seguimento avaliaram a manutenção dos resultados obtidos pela TCC por períodos que variaram entre 10 semanas e 1 ano ${ }^{11,20,21,25-}$ 27,30,33,52.

Ensaios clínicos comparando a TCC com uma lista de espera ou com a TI indicam que, durante o seguimento, há um aumento da freqüência da compulsão alimentar ${ }^{11,20,21}$. Entretanto, esta ainda se mantém em níveis inferiores aos observados no início do TCC ${ }^{11,20,21}$. Wilfley et al. ${ }^{11}$ reportaram que pacientes obesos com TCAP tratados com TCC e TI apresentaram um aumento da compulsão alimentar após o término do tratamento, sendo as taxas de remissão iguais a $72 \%$ na TCC e a $70 \%$ na TI no final do seguimento de 1 ano (e, no pós-tratamento, 82\% na TCC e 74\% na TI). Em contrapartida, foi observada a manutenção das melhoras obtidas nos outros sintomas psicopatológicos característicos do TCAP e uma melhora adicional das dificuldades interpessoais. Neste estudo, durante o seguimento, foi observada uma diminuição de peso estatisticamente significativa, porém não clinicamente significativa, em ambos os modelos de tratamento.

Três estudos controlados randomizados reportaram a manutenção das melhoras obtidas no póstratamento ${ }^{25,27,30}$. Em um ensaio clínico com 114 mulheres obesas com TCAP, Pendleton et al. ${ }^{25}$ reportaram que a taxa de remissão se manteve estável durante o seguimento de 6 meses (61\% no grupo de pacientes submetidos à TCC combinada com exercício físico versus 30\% na TCC isoladamente). Esses autores observaram também que a adição de sessões quinzenais de manutenção, durante os 6 meses subseqüentes ao término da TCC, favoreceu a manutenção da perda de peso. De modo geral, no final do seguimento, os pacientes submetidos à TCC combinada com exercício físico, seguida por manutenção, apresentaram a maior redução de IMC de todos os grupos $(-2,26)$. Adicionalmente, todos os grupos apresentaram manutenção da melhora nos níveis de depressão, estando esta significativamente correlacionada com a melhora da compulsão alimentar. Em outro ensaio clínico, Kenardy et al. ${ }^{30}$ compararam a TCC com um tratamento baseado nos princípios de Rogers e acompanharam durante 3 meses pacientes com TCAP subclínico e diabetes tipo 2. Embora ambos os tratamentos tenham resultado em manutenção das melhoras obtidas no humor e no IMC, observou-se um aumento da taxa de remissão na TCC $(47,1 \%$ no final do tratamento e $58,8 \%$ no final do seguimento) e, em contrapartida, uma piora significativa da compulsão alimentar no tratamento baseado nos princípios de
Rogers (29,4\% no pós-tratamento e 17,6\% no seguimento).

Investigando os resultados obtidos com a associação da TCC com a TC, Agras et al. ${ }^{53}$ avaliaram 93 mulheres obesas com TCAP, 52 semanas após o término do tratamento, e concluíram que houve uma boa manutenção da redução da freqüência da compulsão alimentar (72\% no final do tratamento e $64 \%$ no final do seguimento) e das taxas de remissão ( $41 \%$ no final do tratamento e $33 \%$ no final do seguimento). Entretanto, houve aumento do peso, que retornou aos níveis da linha de base.

A correlação positiva entre freqüência da compulsão e perda de peso, observada ao final do tratamento, também ocorre no seguimento ${ }^{11,25,52}$. Por exemplo, no estudo de Agras et al. ${ }^{53}$, foi reportado que os pacientes que mantiveram remissão durante o seguimento perderam $6,4 \mathrm{~kg}$; os com manutenção parcial perderam 4,1 kg; enquanto os que apresentaram recaída ganharam $0,4 \mathrm{~kg}$ no seguimento.

A análise dos resultados dos estudos citados nesta seção sugere que, ao final de seguimentos de até 1 ano de duração, a compulsão alimentar se mantém em níveis inferiores aos observados no início do tratamento, e há manutenção das melhora obtidas nos níveis de restrição $0^{11,27,34,52}$, na capacidade de inibir o impulso para comer de forma inadequada ${ }^{52}$, nas atitudes disfuncionais associadas à alimentação, ao peso e à forma corporal ${ }^{11,27}$, nos níveis de depressão ${ }^{27,52}$ e nas dificuldades interpessoais ${ }^{11,52}$. Existe, entretanto, uma tendência para o reganho de peso, que está diretamente associado ao fato de o paciente manter episódios de compulsão alimentar no seguimento.

\section{Discussão}

A análise dos resultados dos ensaios clínicos anteriormente descritos sugere que, após a TCC, há uma melhora do funcionamento psicológico geral dos pacientes com TCAP. Foram descritas reduções significativas nos sintomas psicopatológicos característicos do transtorno alimentar e nos sintomas associados. Entretanto, não foi observada diminuição significativa no peso corporal.

Os resultados descritos no presente artigo estão alinhados com os observados numa metanálise realizada por Hay et al. ${ }^{54}$ Nessa metanálise, foi estudada a eficácia de diferentes modelos de psicoterapia no tratamento da BN e de síndromes com compulsão alimentar recorrente. Foram analisados, de forma conjunta, 28 ensaios clínicos com pacientes com bulimia, quatro com pacientes com transtorno alimentar sem outra especificação (um com TCAP) e três com pacientes com TCAP. Nos estudos analisados, foi observado que, após 
a TCC, há uma melhora da compulsão alimentar e de sintomas depressivos, sem alterações no peso corporal. Os autores concluíram que há evidências da eficácia da TCC no tratamento da BN e do TCAP. Entretanto, eles ressaltaram que o número de ensaios clínicos controlados randomizados que avaliaram a eficácia da TCC no TCAP é muito pequeno, sendo necessário realizar mais estudos para formar um corpo de evidências mais sólido.

Embora todos os estudos abertos e controlados tenham demonstrado que há uma redução da freqüência da compulsão alimentar nos grupos tratados, alguns pacientes não melhoram com a TCC ou melhoram apenas parcialmente. Adicionalmente, no longo prazo, foram observados diferentes perfis de resposta, com alguns pacientes apresentando uma piora da compulsão após o término do tratamento e, em contrapartida, outros apresentando melhora ou remissão ao final de períodos de seguimento com duração de até 1 ano $^{30,53}$. No estudo de Agras et al. ${ }^{53}$, dos 31 obesos com TCAP que apresentaram remissão no pós-tratamento, 45\% continuavam em remissão após 1 ano de seguimento, $29 \%$ apresentavam compulsão alimentar não mais de uma vez por semana, e $26 \%$ tinham recaído. Em contrapartida, 25\% dos pacientes que ainda apresentavam compulsão após o término da TCC tiveram remissão no período de seguimento. Esses dados sugerem que, para alguns pacientes, as técnicas testadas nos ensaios clínicos e a duração do tratamento (em geral, 12 sessões) são suficientes. Entretanto, para os não-respondedores, poderia ser considerada a utilização de outros modelos de tratamento ou modificações nos programas da TCC atualmente empregada nos estudos.

Várias alterações nos programas de tratamento foram sugeridas, com o objetivo de melhorar os resultados obtidos com a TCC. Alguns autores sugeriram que um maior número de sessões poderia aumentar a eficácia do tratamento ${ }^{12,25}$. Eldredge et al. ${ }^{12}$ e Pendleton et al. ${ }^{25}$ demonstraram que a adição de 12 sessões aumentou o número de pacientes que melhoraram com a TCC no curto prazo e no período de seguimento de 6 meses. Programas mais longos de tratamento permitiriam um aumento da ênfase dada às técnicas cognitivas que favorecem a reestruturação cognitiva. Isso poderia diminuir a taxa de recaída, uma vez que a reestruturação das crenças disfuncionais associadas à alimentação, ao peso e à forma corporal associa-se a uma melhor manutenção dos resultados no longo prazo ${ }^{14,52}$. Por exemplo, a TC que não inclui reestruturação cognitiva pode auxiliar na redução da compulsão alimentar no curto prazo; contudo, tem eficácia limitada nos outros sintomas psicopatológicos característicos do TCAP, e seus resultados tendem a não se manter a longo prazo $0^{5,8,14,26,45,52,55}$.
Abordagens psicoterápicas que focalizam questões interpessoais ${ }^{11}$ ou que enfatizam o treinamento na regulação dos afetos favorecem reduções na freqüência da compulsão alimentar em obesos com TCAP ${ }^{56,57}$. Na prática clínica geral, a TCC utiliza uma ampla variedade de técnicas para abordar dificuldades interpessoais e estratégias para propiciar que o paciente possa regular afetos. No entanto, esses aspectos foram pouco focalizados nos formatos de tratamento testados nos ensaios clínicos com TCAP. Futuros estudos poderiam avaliar a eficácia de incluir técnicas voltadas para essas questões nos programas de tratamento já existentes. Adicionalmente, devese considerar o fato de que alguns pacientes não melhoram com a adição de técnicas focalizadas em aspectos interpessoais ${ }^{24}$, e outros não melhoram após programas de terapia focalizados no ensino da regulação do humor ${ }^{57}$. Por outro lado, 12 sessões de TCC são suficientes para alguns pacientes e insuficientes para outros. Esses dados, somados, sugerem que os pacientes com TCAP apresentam diferentes perfis de resposta. Ainda não foram encontrados fatores que pudessem predizer de forma confiável o sucesso no tratamento, para que se pudesse adequar o perfil do paciente ao melhor formato de terapia ${ }^{58}$. Wilson ${ }^{58}$ sugeriu que o tratamento poderia ser oferecido no formato de módulos, de acordo com as dificuldades de cada paciente. Inicialmente, seriam utilizadas intervenções mínimas (psicoeducação, manuais de auto-ajuda ou protocolos breves de tratamento) e, posteriormente, seriam adicionados módulos que enfocassem dificuldades específicas para os pacientes que apresentarem pior resposta às intervenções mais breves.

O pouco impacto obtido pela TCC no IMC dos pacientes poderia ser decorrente dos formatos de tratamento utilizados na maioria dos ensaios clínicos. Partindo-se da idéia de que o TCAP e a BN têm características em comum, a proposta inicial de tratamento foi adaptar para o TCAP o modelo terapêutico que se mostrou eficaz no tratamento da BN. Conseqüentemente, não foram inseridas de forma extensa as estratégias dirigidas para a perda de peso, sendo as metas primárias do tratamento a redução da freqüência da compulsão alimentar e a melhora dos outros sintomas psicopatológicos característicos do TCAP. Alguns programas de TCC orientam os pacientes a focalizarem na questão da perda de peso depois de terem obtido controle da compulsão alimentar ${ }^{16,20}$. O pressuposto inicial foi que o foco prematuro na questão do emagrecimento poderia incentivar um aumento da restrição alimentar e que esta seria um fator predisponente para a ocorrência da compulsão alimentar. 
À medida que o perfil dos pacientes com TCAP foi sendo estudado, observou-se que, embora a restrição alimentar possa favorecer a ocorrência de compulsão em alguns pacientes, esse efeito não é observado em todos os casos $^{5,41}$. Esse fato pode ser exemplificado pelos resultados obtidos nos estudos que associam TC com dietas que incentivam a restrição alimentar. Além de ser bem-sucedida na redução do peso corporal ${ }^{5,14,24,26,47}$, a TC pode favorecer algum grau de melhora da compulsão alimentar no curto prazo ${ }^{14,24}$. Adicionalmente, estudos que associaram a TCC a programas estruturados de orientação nutricional e de atividade física observaram perda de peso e melhora da compulsão alimentar ${ }^{25,34,35}$. Esses dados sugerem que a inclusão de estratégias voltadas para a perda de peso não necessariamente se contrapõe ao tratamento dos sintomas psicopatológicos característicos do TCAP e que a TCC pode ampliar suas metas de modo a incluir também o emagrecimento. Devlin ${ }^{59}$ propôs um tratamento com duas linhas de atuação combinadas. Por um lado, são implementadas estratégias comportamentais e cognitivas que focalizam a normalização da alimentação, a eliminação da compulsão alimentar e o emagrecimento. Por outro lado, os pacientes são auxiliados a examinar e reavaliar suas atitudes associadas à aparência, suas crenças sobre valor pessoal e suas idéias supervalorizadas sobre a obesidade. O tratamento objetiva obter uma diminuição da distância entre a faixa de peso sustentável e o peso que o paciente considera aceitável. Implícita a esse programa, está a importância de atingir um equilíbrio entre a efetuação de modificações e a auto-aceitação dos limites individuais.

Um outro aspecto importante em relação aos estudos com TCC no TCAP é que a maior parte dos ensaios clínicos utilizou programas de terapia em grupo. São necessários mais estudos que avaliem a eficácia da TCC quando implementada em formato individual, uma vez que ela torna mais fácil flexibilizar o tratamento, adaptando-o ao perfil do paciente. Outro aspecto pouco estudado nos ensaios clínicos é a inclusão da família nos programas de tratamento ou o estabelecimento de outras redes de apoio social.

Alguns formatos de TCC avaliados nos ensaios clínicos não incluíram estratégias voltadas para a manutenção dos resultados e, naqueles que incluíram, o número de sessões dedicadas ao tema variou entre uma e seis. Entretanto, uma maior ênfase no desenvolvimento de técnicas para prevenção de recaídas poderia aumentar o número de pacientes que consegue manter os resultados obtidos no longo prazo. São necessários mais estudos para identificar a quantidade ideal de apoio posterior ao término do tratamento, sendo eventualmente útil manter sessões espaçadas para reforço do tratamento por períodos longos de tempo.

Há poucos ensaios clínicos avaliando a eficácia da TCC no TCAP ${ }^{54}$, e algumas limitações metodológicas dos estudos analisados devem ser consideradas. $\mathrm{Na}$ maioria dos ensaios clínicos, as amostras são pequenas ${ }^{54}$. Adicionalmente, os resultados da TCC foram avaliados predominantemente em mulheres americanas e européias, com idade entre 18 e 65 anos e IMC maior ou igual a 27, dificultando a generalização dos resultados para outras populações. Além disso, a dependência de álcool ou drogas é um fator de exclusão comum, sendo necessários mais estudos para avaliar os resultados da TCC em pacientes com estas comorbidades. Por último, estudos com seguimentos maiores do que 1 ano permitirão uma melhor avaliação dos efeitos da TCC no longo prazo.

\section{Conclusões}

Os programas de TCC resultam em melhoras significativas nos sintomas psicopatológicos característicos do TCAP, sem reduções significativas no peso corporal. São também observadas melhoras na autoestima, nas dificuldades interpessoais, no humor e na qualidade de vida, além de um aumento do sentimento subjetivo de bem-estar. Entretanto, nem todos os pacientes apresentam boa resposta à TCC, e algumas adaptações nos protocolos de tratamento foram sugeridas, com o objetivo de aumentar o número de respondedores. Dentre as modificações sugeridas, está a inserção de técnicas voltadas para a perda de peso e o aumento da ênfase dada às técnicas para treinamento da regulação do humor e às questões interpessoais. Ainda não está claramente definido o número ideal de sessões, a seqüência em que as técnicas devem ser implementadas, quantas sessões devem ser dedicadas às estratégias comportamentais e quantas devem ser dedicadas às estratégias cognitivas. Adicionalmente, ainda se faz necessária a identificação de fatores preditivos de sucesso no tratamento, para que se possa adaptar protocolos específicos a perfis individuais de resposta. Atingir perdas de peso que se mantenham no longo prazo faz parte do desafio maior de desenvolver métodos eficazes de manutenção de peso para obesos de modo geral.

Há menos estudos sobre a eficácia da TCC no tratamento no TCAP quando comparados aos estudos com BN. Entretanto, dentre os modelos de terapia propostos para o TCAP, a TCC é o modelo com resultados mais bem documentados. De modo geral, a TCC é um método eficaz de tratamento para o TCAP, e nenhum outro modelo terapêutico com o qual tenha sido comparada mostrou ser significativamente mais eficaz. 


\section{Referências}

1. American Psychiatric Association - APA. Diagnostic and statistical manual of mental disorders. 4th ed. Washington (DC): American Psychiatric Association; 1994.

2. Spitzer RL, Devlin M, Walsh BT, Hasin D, Wing R, Marcus M, et al. Binge eating disorder: a multisite field trial of the diagnostic criteria. Int J Eat Disord. 1992;11(3):191-203.

3. Spitzer RL, Yanovski S, Wadden T, Wing R, Marcus M, Stunkard AJ et al. Binge eating disorder: its further validation in a multisite study. Int J Eat Disord. 1993;13(2):137-53.

4. Telch CF, Stice E. Psychiatric comorbidity in women with binge eating disorder: prevalence rates from a non-treatment seeking sample. J Consult Clin Psychol. 1998;66(5):768-76.

5. Marcus MD, Wing RR, Ewing L, Kern E, McDermott M, Gooding W. A double-blind, placebo controlled trial of fluoxetine plus behavior modification in the treatment of obese binge eaters and nonbinge eaters. Am J Psychiatry. 1990;147(7):876-81.

6. Kolotkin RL, Revis ES, Kirkley BG, Janick L. Binge eating in obesity: associated MMPI characteristics. J Consult Clin Psychol. 1987;55(6):872-6

7. Telch CF, Agras WS. Obesity, binge eating and psychopathology: are they related? Int J Eat Disord. 1994;15(1):53-61.

8. Marcus MD, Wing RR, Hopkins J. Obese binge eaters: affect, cognitions and response to behavioral weight control. J Consult Clin Psychol. 1988;56(3):433-9.

9. Yanovski SZ, Nelson JE, Dubbert BK, Spitzer RL. Association of binge eating disorder and psychiatric comorbidity in obese subjects. Am J Psychiatry. 1993;150(10):1472-9.

10. Marchesini G, Solaroli E, Baraldi L, Natale S, Migliorini S, Visani E, et al. Health-related quality of life in obesity: the role of eating behavior. Diabetes Nutr Metab. 2000;13(3):156-64.

11. Wilfley DE, Welch RR, Stein RI, Spurrell EB, Cohen LR, Saelens $\mathrm{BE}$, et al. A randomized comparison of group cognitive-behavioral therapy and group interpersonal psychotherapy for the treatment of overweight individuals with binge-eating disorder. Arch Gen Psychiatry. 2002;59(8):713-21.

12. Eldredge KL, Steward Agras W, Arnow B, Telch CF, Bell S, Castonguay L, et al. The effects of extending cognitive-behavioral therapy for binge eating disorder among initial treatment nonresponders. Int J Eat Disord. 1997;21(4):347-52.

13. Keefe PH, Wyshogrod D, Weinberger E. Agras WS. Binge eating and outcome of behavioral treatment of obesity: a preliminary report. Behav Res Ther. 1984;22(3):319-21.

14. Nauta H, Hospers H, Kok G, Jansen A. A comparison between a cognitive and a behavioral treatment for obese binge eaters and obese non-binge eaters. Behav Ther. 2000;31:441-61.

15. National Institute for Health and Clinical Excellence (NICE). Eating disorders: core interventions in the treatment and management of anorexia nervosa, bulimia nervosa and related eating disorders. Clin. Guideline No. 9. London. Available from: www.nice.org.uk.

16. Fairburn CG, Marcus MD, Wilson GT. Cognitive-behavioral therapy for binge eating and bulimia nervosa: a comprehensive treatment manual. In: Fairburn CG, Wilson GT, eds. Binge eating: nature, assessment and treatment. New york: Guilford Press; 1993. p. 371404.

17. Marcus MD. Adapting treatment for patients with binge-eating disorder. In: Garner DM, Garfinkel PE, eds. Handbook of treatment for eating disorders 2nd ed. New York: The Guilford press; 1997. p. 484-93.

18. Telch CF, Agras WS, Rossiter EM. Group cognitive-behavioral therapy for binge eating disorder: therapist manual. Palo Alto (CA): Stanford University press; 1990.

19. Devlin MJ, Fischer SE. Treatment of binge eating disorder. In: Wonderlich S, Mitchell J, de Zwaan M, Steiger H, editors. Eating disorders review. Part 1. Oxford Radcliffe Publishing; 2005. p. 2741.

20. Telch CF, Agras WS, Rossiter EM, Wilfley D, Kenardy J. Group cognitive behavioral treatment for the nonpurging bulimic: an initial evaluation. J Consult Clin Psychol. 1990;58(5);629-35.

21. Wilfley DE, Agras WS, Telch CF, Rossiter EM, Schneider JA, Cole
AG, et al. Group cognitive-behavioral therapy and group interpersonal psychotherapy for the nonpurging bulimic individual: a controlled comparison. J consult Clin Psychol. 1993;61(2);296-305.

22. Wolff GE, Clark MM. Changes in eating self-efficacy and body image following cognitive-behavioral group therapy for binge eating disorder: a clinical study. Eat Behav. 2001;2(2):97-104.

23. Smith DE, Marcus MD, Kaye W. Cognitive-behavioral treatment of obese binge eaters. Int J Eat Disord. 1992;12(3);257-62.

24. Agras WS, Telch CF, Arnow B, Eldredge K, Detzer MJ, Henderson $\mathrm{J}$, et al. Does interpersonal therapy help patients with binge eating disorder who fail to respond to cognitive-behavioral therapy? J consult Clin Psychol. 1995;63(3);356-60.

25. Pendleton VR, Goodrick GK, Poston WS, Reeves RS, Foreyt JP. Exercise augments the effects of cognitive-behavioral therapy in the treatment of binge eating. Int J Eat Disord. 2002;31(2):172-84.

26. Agras WS, Telch CF, Arnow B, Eldredge K, Wilfley DE, Raeburn $\mathrm{SD}$, et al. Weight loss, cognitive-behavioral and desipramine treatments in binge eating disorder: an addictive design. Behav Ther. 1994;25:209-38.

27. Ricca V, Mannucci E, Mezzani B, Moretti S, Di Bernardo M, Bertelli $\mathrm{M}$, et al. Fluoxetine and fluvoxamine combined with individual cognitive-behaviour therapy in binge eating disorder: a one-year follow-up study. Psychother Psychosom. 2001;70(6):298-306.

28. Devlin MJ, Goldfein JA, Petkova E, Jiang H, Raizman PS, Wolk S, et al. Cognitive behavioral therapy and fluoxetine as adjuncts to group behavioral therapy for binge eating disorder. Obes Res. 2005;13(6):1077-88.

29. Grilo CM , Masheb RM, Wilson GT. Efficacy of cognitive behavioral therapy and fluoxetine for the treatment of binge eating disorder: a randomized double-blind placebo-controlled comparison. Biol Psychiatry. 2005;57(3):301-9.

30. Kenardy J, Mensch M, Bowen K, Green B, Walton J. Group therapy for binge eating in type 2 diabetes: a randomized trial. Diabet Med. 2002;19(3);234-9.

31. Rice LN, Greenberg LS. Patterns of change: intensive analysis of psychotherapy process. New York: Guilford; 1983.

32. Freitas S, Lopes CS, Coutinho W, Appolinario JC. Translation and adaptation into portuguese of the Binge-Eating Scale. Rev Bras Psiquiatr. 2001;23(4):215-20.

33. Porzelius LK, Houston C, Smith M, Arfken C, Fisher E. Comparison of a standard behavioral weight loss treatment and a binge eating weight loss treatment. Behav Ther. 1995;26:119-34.

34. Fossati M, Amati F, Painot D, Reiner M, Haenni C, Golay A. Cognitive-behavioral therapy with simultaneous nutritional and physical activity education in obese patients with binge eating disorder. Eat Weight Disord. 2004;9(2);134-8.

35. Painot D, Jotterand S, Kammer A, Fossati M, Golay A. Simultaneous nutritional cognitive-behavioural therapy in obese patients. Patient Educ Couns. 2001;42(1):47-52.

36. Stunkard AJ, Messick S. The Three-Factor Eating Questionnaire to measure dietary restraint, disinhibition and hunger. J Psychosom Res. 1985;29(1):71-83.

37. Fairburn CG, Cooper Z. The eating disorder examination. In: Fairburn CG, Wilson GT, eds. Binge eating: nature, assessment and treatment. New York: Guilford; 1993. p. 317-60.

38. Garner DM. Eating Disorder Inventory-2. Odessa (FL): Psychological Assessment Resources; 1991

39. Lowe MR, Caputo GC. Binge eating in obesity: toward the specification of predictors. Int J Eat Disord. 1991;10(1):49-55.

40. Laessle RG, Tuschl RJ, Kotthaus BC, Pirke KM. A comparison of the validity of the three scales for the assessment of dietary restraint. J Abnorm Psychol. 1989;98(4):504-7.

41. Wardle J, Waller J, Rapoport L. Body dissatisfaction and binge eating in obese women: the role of restraint and depression. Obes Res. 2001;9(12):778-87.

42. Sarwer DB, Wadden TA, Foster GD. Assessment of body image dissatisfaction in obese women: specificity, severity and clinical significance. J Consult Clin Psychol. 1998;66(4):651-4.

43. Parham ES. Promoting body size acceptance in weight management counseling. J Am Diet Assoc. 1999;99(8):920-5.

44. Beck AT, Ward CH, Mendelson M, Mock J, Erbaugh J. An inventory for measuring depression. Arch Gen Psychiatry. 1961;4:561-71. 
45. Marchesini G, Natale S, Chierici S, Manini R, Besteghi L, Di Domizio $\mathrm{S}$, et al. Effects of cognitive-behavioural therapy on health-related quality of life in obese subjects with and without binge eating disorder. Int J Obes Relat Metab Disord. 2002;26(9):1261-7.

46. Rosenberg M. Conceiving the self. New York: Basic Books; 1979.

47. Horowitz LM, Rosenberg SE, Baer BA, Ureno G, Villasenor VS Inventory of interpersonal problems: psychometric properties and clinical applications. J Consult Clin Psychol. 1988;56(6):885-92.

48. Bradley C. The wellbeing questionnaire. In: Bradley C, ed. Handbook of psychology and diabetes. Sydney: Harwood; 1994.

49. McHorney CA, Ware JE, Raczek AE. The MOS 36-Item Short-Form Health Survey (SF-36):II. Psychometric and clinical tests of validity in measuring physical and mental health constructs. Med Care. 1993;31(3):247-63.

50. Brownell KD. The LEARN program for weight control. Philadelphia: University of Pennsylvania; 1989.

51. Wilfley DE, Frank MA,Welch R, Spurrell EB, Rounsaville BJ. Adapting interpersonal psychotherapy to a group format (IPT-G) for binge eating disorder: toward a model for adapting empirically supported treatments. Psychotherapy Res. 1998;8:379:91.

52. Nauta H, Hospers H, Jansen A. One-year follow-up effects of two obesity treatments on psychological well-being and weight. $\mathrm{Br} \mathrm{J}$ Health Psychol. 2001;6(3):271-84.

53. Agras WS, Telch CF, Arnow B, Eldredge K, Marnell M. One-year follow-up of cognitive-behavioral therapy for obese individuals with binge eating disorder. J consult Clin Psychol. 1997;65(2):343-7.

54. Hay PJ, Bacaltchuk J, Stefano S. Psychotherapy for bulimia nervosa and binging (Cochrane Review). In: The Cochrane Library. Oxford: Update Software. 2005;2.

55. Wadden TA, Foster GD, Letizia KA. One-year behavioral treatment of obesity: comparison of moderate and severe caloric restriction and the effects of weight maintenance therapy. J. Consult Clin Psychol. 1994;62(1):165-71.

56. Telch CF, Agras WS, Linehan MM. Dialectical behavior therapy for binge eating disorder. J Consult Clin Psychol. 2001;69(6):1061-5.

57. Safer DL, Lively TJ, Telch CF, Agras WS. Predictors of relapse following successful dialectical behavior therapy for binge eating disorder. Int J Eat Disord. 2002;32(2):155-63.

58. Wilson GT. Psychological treatment of binge eating and bulimia nervosa. J Ment Health. 1995;4(5):451-8.

59. Devlin MJ. Binge-eating disorder and obesity. A combined treatment approach. Psychiatr Clin North Am. 2001;24(2):325-35. 